\title{
Extending Mirror Conjecture to Calabi-yau with Bundles
}

\section{Citation}

VAFA, CUMRUN. 1999. "EXTENDING MIRROR CONJECTURE TO CALABI-YAU WITH BUNDLES." Communications in Contemporary Mathematics 1 (1): 65-70. https://doi.org/10.1142/ s0219199799000043.

\section{Permanent link}

http://nrs.harvard.edu/urn-3:HUL.InstRepos:41385059

\section{Terms of Use}

This article was downloaded from Harvard University's DASH repository, and is made available under the terms and conditions applicable to Other Posted Material, as set forth at http:// nrs.harvard.edu/urn-3:HUL.InstRepos:dash.current.terms-of-use\#LAA

\section{Share Your Story}

The Harvard community has made this article openly available.

Please share how this access benefits you. Submit a story.

Accessibility 
HUTP-98/A033

hep-th/9804131

\title{
Extending Mirror Conjecture to Calabi-Yau with Bundles
}

\author{
Cumrun Vafa \\ Lyman Laboratory of Physics \\ Harvard University \\ Cambridge, MA 02138, USA
}

\begin{abstract}
We define the notion of mirror of a Calabi-Yau manifold with a stable bundle in the context of type II strings in terms of supersymmetric cycles on the mirror. This allows us to relate the variation of Hodge structure for cohomologies arising from the bundle to the counting of holomorphic maps of Riemann surfaces with boundary on the mirror side. Moreover it opens up the possibility of studying bundles on Calabi-Yau manifolds in terms of supersymmetric cycles on the mirror.
\end{abstract}

April 1998 


\section{Introduction}

Mirror symmetry, which was conjectured as a generalization of $R \rightarrow 1 / R$ duality for Calabi-Yau compactifications [1] 2] has played a major role in our understanding of dynamical issues in string theory. At the level of string perturbation theory, the concrete examples of mirror pairs [3] was shown to result in a deeper understanding of sigma model instantons [ [ 1 . Non-perturbatively mirror symmetry also plays a key role in the geometric engineering approach to constructing quantum field theories. The construction of mirror pairs has been generalized using toric methods [5] and some proposals exist as to how one may derive these constructions starting from $R \rightarrow 1 / R$ duality [6] [7] [8] [9]. The basic idea is to view in a certain degenerate limit the Calabi-Yau $n$-fold as locally a fiber space with fiber an $n$ dimensional real torus, and applying T-daulity fiber by fiber. The purpose of this note is to extend the mirror conjecture to situations including bundles over CalabiYau. The basic idea of this extended conjecture was motivated by the topological open and closed strings on Calabi-Yau threefolds [10][11] and was in fact discussed in a preliminary form in [12]. The sharpened version of the present conjecture is motivated by the recent advances in our understanding of D-branes [13]. See also the related works [14] [15] [16].

Bundles over Calabi-Yau 3-folds have phenomenological applications for constructing $N=1, d=4$ vacua for heterotic string. In this context they lead to $(2,0)$ sigma model on the worldsheet and one could attempt to construct a mirror in this context. Some proposals have been made in this setup [17] [18]. This is not what we will be considering in this paper. We will concentrate on bundles in the context of wrapped D-branes over CalabiYau manifolds and discuss their mirror. Some of the constructions are most natural for the Calabi-Yau threefold which will also be the main focus of this paper, though generalizations are straight-forward for any $n$-fold Calabi-Yau and some of our general discussion is in that context.

\section{Basic Review of Standard Mirror Symmetry}

We start with a Calabi-Yau 3-fold $M$ and its mirror pair $W$. Very roughly speaking $M$ and $W$ are fiber spaces with fiber $T^{3}$ and the main difference between them is that in one case the radii of $T^{3}$ are inverted. This is related to proposal of [6] for viewing the mirror Calabi-Yau as the moduli of a supersymmetric $T^{3}$ cycle. This view of the mirror pair has been developed in [9] with the result that one can give an intuitive physical explanation of constructing mirror pairs using dual toric data [5]. 
One of the basic consequences of mirror symmetry is that curve counting becomes very simple. Let $t^{i}$ parameterize the Kahler moduli of $M$ relative to a basis of 2 -cycles $\left[C_{i}\right]$,

i.e. $t_{i}=\int_{C_{i}} k$ where $k$ is the Kahler form. Moreover let $q_{i}=e^{-t_{i}}$. Let $d^{\left\{n_{l}\right\}}$ denote the number of holomorphic maps from the sphere to Calabi-Yau, whose image is in $\sum n_{i}\left[C_{i}\right]$. On the other hand consider $W$ and the unique (up to overall scale) holomorphic 3-form on it $\Omega$. Then the basic mirror conjecture in particular implies that

$$
\sum n_{i} n_{j} n_{k} d^{\left\{n_{l}\right\}} \frac{\prod_{l} q_{l}^{n_{l}}}{\left(1-\prod_{l} q_{l}^{n_{l}}\right)}=\int_{W} \partial_{i} \partial_{j} \partial_{k} \Omega \wedge \Omega
$$

for suitable choice of coordinates on moduli of complex structure of $W$ (known as special coordinates) and normalization of $\Omega$. The denominator in the left-hand side of the above formula comes from contribution of multi-wrapped instantons in $M$.

\section{Extension of Mirror Conjecture}

In this section we extend the mirror conjecture to the case of Calabi-Yau with bundles on top of it. Let us be physically concrete. Consider a Calabi-Yau n-fold $W$ with $N$ copies of $D_{2 n}$ branes wrapped over it (here we are considering Euclidean branes and so this is conventionally denoted by $D_{2 n-1}$ Euclidean brane-here it is more natural to denote it by $\left.D_{2 n}\right)$. This gives rise to a $U(N)$ gauge bundle on top of Calabi-Yau. The condition to preserve supersymmetry translates to having a stable bundle over $W$ which we will henceforth assume. Moreover the $c_{1}, c_{2}, \ldots, c_{n}$ chern classes of the bundle can be interpreted as the number (more precisely homology class) of $D_{2 n-2}, D_{2 n-4}, \ldots, D_{0}$ branes bound to the $D_{2 n}$ brane [19] [20]. The bundle should be non-degenerate if there is no common multiple to $N, c_{1}, \ldots, c_{n}$. What should we expect the mirror to be?

Given that the mirror symmetry is at least morally T-duality on each $T^{n}$ fiber, and T-duality on D-branes exchanges Dirichlet and Neumann boundary conditions the mirror of $N$ copies of $D_{2 n}$ brane will be an $n$-dimensional real submanifold of $M$. Moreover if the bundle over $W$ is stable, as we assumed, then the mirror should be a supersymmetric $n$ cycle (Lagrangian relative to the Kahler 2 -form and with minimal area). Moreover the homology class of the $n$-cycle is completely fixed by $c_{i}$ of the bundle as there is a map from each $(k, k)$ class of $M$ to an element of $H_{n}(W)$ (see in particular [21] which is very much in the spirit of the present discussion). In particular the homology class of $C, H_{n}(C)$, is the mirror image of $\sum_{i}\left[c_{i}\right]$ (where we include $c_{0}$ in this sum). 
Let us assume that the bundle $V$ is non-degenerate over $W$ and so its mirror is a smooth $n$-cycle $C \subset M$. Apart from the above relations between $V$ and $C$ there is one more important relation: Since the resulting physics should be indistinguishable, this implies that the moduli space of stable bundles $V$ with fixed $c_{i}$, whose complex dimension is given by $H^{1}(E n d V)$ should be equal to the dimension of moduli of the supersymmetric $n$-cycle $C$. We now argue that this in turn is equal to $H^{1}(C)$. To see this note that on the $D_{n}$ brane wrapped over $C$ we have a $U(1)$ bundle. Thus the dimension of moduli of flat wilson lines is $b^{1}(C)$. This, on the other hand should be paired with another moduli to become the phase of a complex moduli (similar to how the B-field and the real Kahler class combine). We thus conclude that the complex moduli of the supersymmetric cylce $C$, as far as its embedding in $M$ is concerned should be $H^{1}(C)$. This is indeed the case, as the detailed analysis of [6] demonstrates. Thus the complex dimension of the moduli space of the supersymmetric cycle $C$ is $b^{1}(C)$ and we thus have an identification

$$
H^{1}(E n d V, W)=H^{1}(C, M)
$$

In fact more should be true, because the whole complex should map to the corresponding mirror, i.e. we should expect for all $k$

$$
H^{k}(E n d V, W)=H^{k}(C, M) .
$$

It is natural to choose "special" coordinates for the moduli space of supersymmetric $C$ cycle as follows: Let

$$
\left\{\gamma_{j}\right\}=H_{1}(C)
$$

denote a basis for the 1-cycles of $C$. Let $k$ denote the Kahler class of $M$, which we decompose in terms of an integral basis $\left\{k_{i}\right\}$ of $H^{2}(M, \mathbf{Z})$

$$
k=\sum_{i=1}^{h^{1,1}(M)} t_{i} k_{i}
$$

Let us further assume that $H_{1}(M)=0$ (as is the case other than for tori). Then each $\gamma_{j} \in H_{1}(C)$ is contractible in $M$ and we choose a minimal area disk $D_{j} \subset M$, which is holomorphic, whose boundary is $\gamma_{j}$, i.e.

$$
\partial D_{j}=\gamma_{j}
$$


Define a real coordinate for the moduli of the supersymmetric cycle $C$ by

$$
r_{j}=\int_{D_{j}} k
$$

i.e., the area of a minimal disk. Note that this will not depend on the choice of the representative $\gamma_{j}$, because if we deform it to another 1-cycle $\gamma_{j}^{\prime}$, and consider $\Delta$ which is a 2 dimensional subspace in $C$ with $\partial \Delta=\gamma_{j}-\gamma_{j}^{\prime}$, then the difference in "area" of the disks is $\int_{\Delta} k=0$, because $k$ restricted to $C$ is zero (recall $C$ is Lagrangian relative to $k$ ).

Now, let the $U(1)$ holonomy around $\gamma_{j}$ be denoted by $a_{j}$. We combine $r_{j}$ and $a_{j}$ to a "special" complex coordinate by

$$
\tilde{r}_{j}=r_{j}+i a_{j}
$$

It is also natural to define the complex algebraic coordinates

$$
\tilde{q}_{j}=\exp \left(-\tilde{r}_{j}\right)
$$

Note that we now have as many complex coordinates for the moduli of supersymmetric cycle $C$ as $b^{1}(C)$. Note that any other disk with boundary $\gamma_{j}$ will have an area

$$
A_{j}=r_{j}+\sum n_{i} t_{i}
$$

with positive integers $n_{i}$. This follows because the difference between two disks bounding $\gamma_{j}$ is a closed 2-cycle in $W$ and so $\int k_{i}$ over it gives the positive integer $n_{i}$.

Finally we apply this to Calabi-Yau threefold case and write a formula generalizing (2.1). In fact it is relatively straight-forward to come up with the generalization. The basic idea is that (2.1) related computation of one topological theory to another for the closed string sector and we are after the open string sector of it. The general structure of the open string field theory in the target space has been studied in [10]. On the complex side, $W$, we have a holomorphic version of Chern-Simons theory with action

$$
S=\int_{W} \Omega \wedge \operatorname{Tr}\left(A \bar{\partial} A+\frac{2}{3} A^{3}\right)
$$

where $A$ is a holomorphic (stable) $U(N)$ gauge connection which is a $(0,1)$ form on CalabiYau threefold $W$ with values in the adjoint representation of $U(N)$. On the $M$ side we have the usual Chern-Simons theory corrected by sigma model instantons, i.e.,

$$
S=\int_{C} \operatorname{Tr}\left(\mathcal{A} d \mathcal{A}+\frac{2}{3} \mathcal{A}^{3}\right)+\text { Instanton corrections }
$$


where the instanton corrections involve summing over holomorphic maps from Riemann surfaces with boundaries, where boundary of the Riemann surface is mapped to $C$, weighed by $\exp (-A)$ as well as with insertion of Wilson line on $C$. The specific generalization of (2.1) will correspond to computing the disk worldsheet with three insertions of the $H^{1}$ fields of $C$ at the boundary (to rigidify the disk). Setting these two contributions from the two gauge theories equal will result in generalization of (2.1). The equality results in

$$
\int_{W} \operatorname{Tr}\left[\delta_{r} A \delta_{s} A \delta_{p} A\right] \wedge \Omega=\sum_{m_{j}, n_{i}} d_{\left\{m_{j}\right\}}^{\left\{n_{i}\right\}}(r, s, p) \prod_{j=1}^{b^{1}(C)} \tilde{q}_{j}^{m_{j}} \prod_{i=1}^{h^{1,1}(M)} q_{i}^{n_{i}}
$$

where $d_{\left\{m_{j}\right\}}^{\left\{n_{i}\right\}}(r, s, p)$ denotes the number of holomorphic maps (up to a sign [10]) from a disk to $M$ where the boundary of the disk is mapped to $C$ satisfying the following conditions: First, the class of this boundary curve in $H_{1}(C)$ is

$$
\partial D=\sum_{j} m_{j}\left[\gamma_{j}\right]
$$

Moreover, the class of $D-\sum m_{j} D_{j}$, with $D_{j}$ defined above, which is a closed 2-cycle in $M$, is denoted by integers $n_{i}$ (relative to the integral basis for $H_{2}(M)$ ). Furthermore we require that three marked points on the boundary of the disk get mapped in a definite cyclic order to a point in specific 2-cycles $C^{r}, C^{s}, C^{p} \subset C$ in that order, which are Poincare dual to $\gamma_{r}, \gamma_{s}, \gamma_{p}$ in $C$. Note that just as in the usual mirror symmetry, the number of such maps, in case there is a family of them, is to be understood as the Euler class of some appropriate bundle on moduli space. Also one expects a similar modification due to multi-wrapped disks, somewhat modifying the above formula as in the usual case of mirror symmetry. A special case of the above relation is when $m_{j}=0=n_{i}$ in which case on the Chern-Simons side one is getting the classical triple intersection of the 2-cycles $(r, s, p)$ on C.

The left-hand side of (3.5) which involves derivatives of the $(0,1)$-form connection $A$ relative to moduli of stable bundles in directions $\mathrm{r}, \mathrm{s}$ and $\mathrm{p}$ is a natural generalization of variation of Hodge structure in the present context. Its structure in the above equation arises simply by considering a three point function of the topological open string B-model, and mapping it by mirror symmetry to the corresponding three point function of the topological open string A-model. Note that the left-hand side of (3.5) will depend on $q_{i}$ from the underlying complex structure of Calabi-Yau and the fact that the holomorphic 
connection $A$ knows about it, as well as $\Omega$ in (3.5). It also depends, of course on the moduli of bundle which we are denoting by $\tilde{q}_{j}$.

Here we have only compared the contribution of one open string worldsheet on the two sides. One can of course do this for arbitrary worldsheet geometries, just as in the closed string case [11]. For example, just as in closed string case [11] we would expect that if we considered the annulus instead of disk, on the $W$ side we would be computing the Ray-Singer torsion of the bundle $V$ over $W$ and on the $M$ side the holomorphic maps from annulus to $M$ with boundary being mapped to pairs of curves in $C$. Clearly it would be extremely interesting to work out some explicit examples in detail.

So far we have emphasized the role of the stable bundle in solving an enumerative algebraic problem on the mirror. One can also use this in the opposite direction. Namely, instead of thinking about bundles on the original manifold we might as well study middimension supersymmetric cycles on the mirror. In the context of bundles on $K 3$ this results in a surface on the mirror and the study of this curve is equivalent to studying the original bundle. In this context this is well known to mathematicians and in fact has been used in physical applications [22] [23]. The generalization of this relation between bundles and cycles on the mirror in higher dimensions which we have found here is mathematically new.

We would like to thank Michael Bershadsky, Brian Greene, Maxim Kontsevich and Eric Zaslow for valuable discussions.

This research is supported in part by NSF grant PHY-92-18167. 


\section{References}

[1] W. Lerche, C. Vafa and N.P. Warner, Nucl. Phys. B324 (1989) 427.

[2] L. Dixon, unpublished.

[3] B. Greene and M.R. Plesser, Nucl. Phys. B338 (1990) 15.

[4] Candelas et. al., Nucl. Phys. B359 (1991) 21.

[5] V. Batyrev, J. Alg. Geom. 3 (1994) 493.

[6] A. Strominger, S.-T. Yau and E. Zaslow, Nucl. Phys. B479 (1996) 243.

[7] D. Morrison, Proc. European Algebraic Geometry Conference (Warwick, 1996), alggeom/9608006.

[8] M. Gross and P.M.H. Wilson, alg-geom/9608004.

[9] N.C. Leung and C. Vafa, hep-th/9711013

[10] E. Witten, hep-th/9207094.

[11] Bershadsky et. al., Comm. Math. Phys. 165 (1994) 311.

[12] C. Vafa, Talk given at IAS, Spring 1994.

[13] J. Polchinski, Phys. Rev. Lett. 75 (1995) 4724.

[14] M. Kontsevich, Proceedings of the 1994 ICM I, Birkäuser, Zürich, 1995, p. 120; alggeom/9411018.

[15] K. Fukaya, The Proceedings of the 1993 GARC Workshop on Geometry and Topology, H.J. Kim, ed., Seoul National University.

[16] A. Polishchuk and E. Zaslow, math.AG/9801119.

[17] J. Distler and S. Kachru, Nucl. Phys. B442 (1995) 64.

[18] E. Sharpe, hep-th/9804066.

[19] M. Douglas, hep-th/9512077.

[20] M. Li, Nucl. Phys. B460 (1996) 351.

[21] H. Ooguri, Y. Oz and Z. Yin, Nucl. Phys. B477 (1996) 407.

[22] R. Friedman, J. Morgan and E. Witten, Comm. Math. Phys. 187 (1997) 679.

[23] M. Bershadsky, A. Johansen, T. Pantev and V. Sadov, Nucl.Phys. B505 (1997) 165. 